\title{
Effect of Neoclassical Transport Optimization on Electron Heat Transport in Low-collisionality LHD plasmas
}

S. MURAKAMI, H. YAMADA ${ }^{1)}$, A. WAKASA ${ }^{2)}$, H. INAGAKI $^{1)}$, K. TANAKA $^{1)}$,

K. NARIHARA ${ }^{1)}$, S. KUBO ${ }^{1)}$, T. SHIMOZUMA ${ }^{1)}$, H. FUNABA ${ }^{1)}$, J.MIYAZAWA ${ }^{1)}$,

S. MORITA ${ }^{1)}$, K. IDA $^{1)}$, S. SAKAKIBARA ${ }^{1)}$, K.Y. WATABNABE ${ }^{1)}$, M. YOKOYAMA ${ }^{11}$,

H. MAASSBERG ${ }^{3)}$, C.D. BEIDLER ${ }^{3)}$, and LHD EXPERIMENTAL GROUP

Department of Nuclear Engineering, Kyoto University, Kyoto 606-8501, Japan

${ }^{1}$ National Institute for Fusion Science, Toki, Gifu 509-5292, Japan

${ }^{2)}$ Graduate School of Engineering, Hokkaido University, Sapporo 060-8628, Japan

${ }^{3)}$ Max-Planck-Institut fuer Plasmaphysik, EURATOM Ass., D-17491 Greifswald, Germany

This manuscript is submitted with reference to the 15th International Stellarator Workshop.

\section{SADAYOSHI MURAKAMI}

Department of Nuclear Engineering, Kyoto University, Kyoto 606-8501, Japan

E-mail: murakami@nucleng.kyoto-u.ac.jp～Phone \& Fax: +81-75-753-5825

Total number of pages : 23 Tables: 0 Figures: 9 


\title{
Effect of Neoclassical Transport Optimization on Electron Heat Transport in Low-collisionality LHD plasmas
}

\author{
S. MURAKAMI, H. YAMADA, A. WAKASA, H. INAGAKI, K. TANAKA, \\ K. NARIHARA, S. KUBO, T. SHIMOZUMA, H. FUNABA, J.MIYAZAWA, \\ S. MORITA, K. IDA, S. SAKAKIBARA, K.Y. WATABNABE, M. YOKOYAMA, \\ H. MAASSBERG, C.D. BEIDLER, and LHD EXPERIMENTAL GROUP
}

\begin{abstract}
.
Electron heat transport in the low-collisonality ECH plasma is investigated to clarify the effect of neoclassical transport optimization on the thermal plasma transport in LHD. Five configurations are realized by shifting the magnetic axis position in major radius: $3.45 \mathrm{~m}, 3.53 \mathrm{~m}, 3.6 \mathrm{~m}, 3.75 \mathrm{~m}$ and $3.9 \mathrm{~m}$. A clear effective helical ripple (which is a quantitative measure of the neoclassical transport optimization) dependency on the enhancement factor of the global energy confinement relative to ISS95 is observed. Local heat transport analyses show a higher electron temperature and a lower heat transport in the neoclassical transport optimized configuration at half the minor radius. The comparisons of the experimental total heat fluxes with that of the neoclassical transport by DCOM/NNW suggests that the neoclassical transport plays a significant role in the heat transport and that the neoclassical transport optimization is effective in improving the plasma confinement in the low-collisionality LHD plasma.
\end{abstract}




\section{Introduction}

The shift of the magnetic axis alters the magnetic field configuration in flux coordinates in LHD. Inward Shift of the magnetic axis from $R_{a x}=3.75 \mathrm{~m}$ ("standard" configuration) to $R_{a x}=3.6 \mathrm{~m}$ conforms to a " $\sigma$-optimized" field[1], where the neoclassical transport would be significantly improved relative to a standard heliotron configuration. A further inward shift of the magnetic axis increases the toroidal mirror term in addition to the two side band terms.

Recent numerical studies of neoclassical transport in the LHD configuration have shown an optimum configuration with respect to $1 / v$ transport when the magnetic axis has a major radius of $3.53 \mathrm{~m}[2]$. In this case, the effective helical ripple is very small, remaining below $2 \%$ inside $4 / 5$ of the plasma radius. Also, in this configuration, the deviation of trapped particle orbit from magnetic surfaces is very small and a good confinement of energetic particle is obtained[3]. The effect of neoclassical transport optimization on the energetic ion confinement has been shown experimentally in LHD[4].

The effective helical ripple for $1 / \nu$ transport, $\varepsilon_{\text {eff }}$, is evaluated by the relation between the transport coefficients, $\mathrm{D}_{1 / v}$, and collision frequency, $v$, in the $1 / v$ regime as

$$
\varepsilon_{\text {eff }}=\left(\frac{9 \sqrt{2} \pi}{16} \frac{v}{v_{d}^{2}} D_{1 / v}\right)^{\frac{2}{3}},
$$

where $\mathrm{v}_{\mathrm{d}}\left(=\kappa / \mathrm{qR}_{0} \mathrm{~B}_{0}\right)$ is the radial drift velocity and $\kappa$ is the kinetic energy. Figure 1 shows the $\varepsilon_{\text {eff }}$ at $r / a=2 / 3$ as a function of the axis position in a major radius. We can see a strong reduction of $\varepsilon_{\text {eff }}$ by inward shift and the obtained value is about $1 \%$ at $R_{a x}=3.53 \mathrm{~m}$. A further inward shift of magnetic axis deteriorates an optimized configuration and the $\varepsilon_{\text {eff }}$ increases again $\left(R_{a x}=3.45 \mathrm{~m}\right)$. These facts indicate that an appropriate inward shift of the magnetic axis can optimize the LHD configuration to a level typical of so-called "advanced stellarators". 
Therefore it is an important issue for the LHD experiment to clarify the effect of configuration optimization on the plasma confinement in a helical system.

The configuration dependency on the plasma confinement in LHD has been investigated in the NBI heated plasma[5-7]. It is found that the global confinement is better in the inward shifted configuration $\left(R_{a x}=3.60 \mathrm{~m}\right.$. $)$ than that of the standard configuration $\left(R_{a x}=3.75 \mathrm{~m}\right)$, and that the local heat and particle transport are dominated by the anomalous transport rather than the neoclassical transport. These experiments have been performed with the density $n_{e} \geq 2.0 \times 10^{19} \mathrm{~m}^{-3}$, in which the collision frequency is relatively high (roughly the plateau regime of the neoclassical transport). Therefore the neoclassical transport effect would be relatively small. If we consider the reactor relevant plasma we have to study less collisional regime, where the neoclassical transport would play an important role.

The transport analysis in the low-collisionality regime has been done in the ECH plasma of LHD[8]. In that experiment, however, a clear effect of the neoclassical transport optimization has not been observed due to the limited number of plasma discharges and magnetic configurations. The available magnetic configuration was limited by the ECH resonance condition because the full magnetic field, $B \sim 3 \mathrm{~T}$, was used.

In this paper the electron heat transport in the low-collisonality plasma (the long-mean-free-path regime; $1 / v$ regime) is analyzed to clarify the effect of configuration optimization on the thermal plasma transport in the LHD. We set the magnetic field strength to $1.5 \mathrm{~T}$ at the magnetic axis and a wide range of magnetic configurations can be investigated. Five typical configurations of LHD are considered; i.e. $R_{a x}=3.45 \mathrm{~m}, 3.53 \mathrm{~m}, 3.6 \mathrm{~m}, 3.75 \mathrm{~m}$ and 3.9m. The ECH heat deposition profiles are distributed inside of $\mathrm{r} / \mathrm{a}=0.3$ in these configurations, and, thus, the total heat flux should be equal to the ECH heating power at the 
outside of the deposition region, $r / a>0.3$ because we apply only $\mathrm{ECH}$ as a heat source in this experiment.

The neoclassical transport is analyzed applying a neural network database of neoclassical transport in LHD by $\mathrm{DCOM} / \mathrm{NNW}[9,10]$. The role of the neoclassical transport is investigated comparing the experimental heat fluxes with that by the neoclassical transport.

In section 2 we show the experimental results about configuration dependencies of the global energy confinement and the local transport in the low-collisionality LHD plasma. The comparisons of the heat flux with the values estimated by the neoclassical transport predictions are shown in section 3. The conclusions are in section 4.

\section{Optimization effect on heat transport in LHD}

The electron heat transport in the low-collisonality plasma is investigated in the ECH plasma of LHD. We considered five typical configurations; $R_{a x}=3.45 \mathrm{~m}, 3.53 \mathrm{~m}, 3.6 \mathrm{~m}, 3.75 \mathrm{~m}$ and $3.9 \mathrm{~m}$. The shift of the magnetic axis alters the magnetic field configuration in flux coordinates, $B=\sum_{m, n} B_{m, n}(\psi) \cos (m \vartheta-n \zeta)$ where $B_{m, n}$ represents the Fourier components of the magnetic field with the poloidal mode number, $m$, and the toroidal mode number, $n$, in Boozer coordinates. In the $R_{a x}=3.75 \mathrm{~m}$ case there are two dominant components, the main helical curvature term, $\mathrm{B}_{2,10}$ and the toroidal curvature term, $\mathrm{B}_{1,0}$, Shifting the magnetic axis inwards to $R_{a x}=3.6 \mathrm{~m}$, two side bands of the main helical curvature term, $\mathrm{B}_{1,10}$ and $\mathrm{B}_{3,10}$ increase and their amplitudes become comparable to that of $\mathrm{B}_{1,0}$. A further inward shift of the magnetic axis increases the toroidal mirror term, $\mathrm{B}_{0,10}$, in addition to the two side band terms for the $R_{a x}=3.53 \mathrm{~m}$ case and, then, the optimum magnetic shift position for the neoclassical 
transport is obatined.

If the neoclassical transport plays a role on the plasma confinement a highest confinement is expected in the $R_{a x}=3.53 \mathrm{~m}$ configuration. In order to study the configuration dependency of the electron heat transport the magnetic field strength, $\mathrm{B} \sim 1.5 \mathrm{~T}$, is used so that we can operate the experiment in these five configurations. We set the magnetic field strength satisfying the ECH resonance condition at the magnetic axis and the ECH $(\mathrm{f} \sim 84 \mathrm{GHz}, \mathrm{P} \sim 880 \mathrm{kw})$ heat deposition profiles are distributed inside of $\mathrm{r} / \mathrm{a}=0.3$ in these configurations. We use only ECH as a heating source of plasma and, thus, the experimental total heat flux should be equal to the ECH heating power outside of the deposition region, $\mathrm{r} / \mathrm{a}>0.3$.

Figure 2 shows a typical time development of the ECH discharge in the LHD $\left(R_{a x}=3.60 \mathrm{~m}\right)$. We started up the plasma with ECH heating and, then, the line averaged density by FIR measurement increased to $1.25 \times 10^{19} \mathrm{~m}^{-3}$. Then a quasi-steady state was obtained about $0.4 \mathrm{~s}$ after the plasma start up. The obtained stored energy became about 100kJ in this discharge.

We, first, study the effect of neoclassical transport optimization on the global energy confinement in the low-collisionality LHD plasma. Figure 3 shows the enhancement factor of the global energy confinement time relative to the ISS95 scaling law[11] as a function of the averaged line density. We can see a clear configuration dependency on the enhancement factors. The highest enhancement factor is obtained in the $R_{a x}=3.53 \mathrm{~m}$ configuration and the lowest is in the $R_{a x}=3.9 \mathrm{~m}$ configuration. Comparing the effective helical ripple in Fig. 1 we can find that the enhancement factor shows an inverse dependence on the effective helical ripple. This result indicates that the global energy confinement is improved by the neoclassical transport optimization in the low-collisionality LHD plasma. It is also found that the enhancement factor deteriorates as the density decreases below $1.0 \times 10^{19} \mathrm{~m}^{-3}$ in all 
configurations.

Next we study the local heat transport in the four configurations; $R_{a x}=3.53 \mathrm{~m}, 3.6 \mathrm{~m}, 3.75 \mathrm{~m}$ and $3.9 \mathrm{~m}$. We plot the electron temperature at $r / a=0.5$ as a function of the line averaged density in Fig. 4; a) $R_{a x}=3.53 \mathrm{~m}$, b) $R_{a x}=3.6 \mathrm{~m}$, c) $R_{a x}=3.75 \mathrm{~m}$ and d) $R_{a x}=3.9 \mathrm{~m}$. The electron temperature profiles are measured by ECE and Thomson scattering measurements and both results show relatively good agreements. The ECE measurement results are used in this analysis due to the high time resolution of ECE. The density profiles are measured by FIR and almost flat density profiles are observed in this experiment, which is a typical one of the $\mathrm{ECH}$ plasma in LHD.

It is found that a highest electron temperature is obtained in the $R_{a x}=3.53 \mathrm{~m}$ configuration and that a lowest one is in the $R_{a x}=3.9 \mathrm{~m}$ configuration. This is consistent with the tendency of the global energy confinement shown previously. Interestingly the electron temperature increases rapidly compared with $1 / n$ when the density decreases to less than $0.5 \times 10^{19} \mathrm{~m}^{-3}$ in the $R_{a x}=3.75 \mathrm{~m}$ and $3.90 \mathrm{~m}$ configurations. This suggests the change of the electron temperature dependence of the heat transport in the lower density region in these configurations.

In order to study the heat transport coefficient we simply evaluate the effective heat transport coefficient, $\chi_{\text {eff }}$, defined by

$$
\chi_{e f f}(r)=-\frac{P_{E C H}}{V^{\prime}(r) n \nabla T_{e}(r)},
$$

where $P_{E C H}$ and $V(r)$ are the total $\mathrm{ECH}$ heating power and the volume at minor radius $r$, respectively. The more detail analysis of heat transport needs the ion temperature profile but we did not have an ion temperature profile measurement in this experiment. Only a central ion temperature is measured by Ar spectrum measurement.

Figure 5 shows the effective heat transport coefficient at $\mathrm{r} / \mathrm{a}=0.5$ as a function of the 
electron temperature for four configurations. The effective heat transport is large, $\chi_{\text {eff }} \sim 10 \mathrm{~m}^{2} / \mathrm{s}$, even for the low electron temperature in the $R_{a x}=3.9 \mathrm{~m}$ configuration. A lower $\chi_{\text {eff }}$ value is obtained in the $R_{a x}=3.53 \mathrm{~m}$ configuration than that in the $R_{a x}=3.6 \mathrm{~m}$ configuration. We can also see that the temperature derivative of $\chi_{\text {eff }}$ gradually increases as the electron temperature increases except the $R_{a x}=3.9 \mathrm{~m}$ configuration, where the gradient is almost constant and no gradual change of the $\chi_{\text {eff }}$ gradient is observed.

Next we see the local heat transport near the edge region $(r / a \sim 0.75)$. Figure 6 shows the electron temperature as a function of the line averaged density, a) $R_{a x}=3.53 \mathrm{~m}$, b) $R_{a x}=3.6 \mathrm{~m}, \mathrm{c}$ ) $R_{a x}=3.75 \mathrm{~m}$ and d) $R_{a x}=3.9 \mathrm{~m}$. The minor radius is at $r / a=0.75$ except the $R_{a x}=3.90 \mathrm{~m}$ configuration where no ECE line exists out side of $r / a=0.65$. We can find that the electron temperature values are similar for the $R_{a x}=3.53 \mathrm{~m}$ and $3.6 \mathrm{~m}$ configurations and that the values are lower in the $R_{a x}=3.75$ and $3.90 \mathrm{~m}$ configurations. The rapid increase of the electron temperature with decreasing density can be seen only in the $R_{a x}=3.9 \mathrm{~m}$ configuration at this minor radius. The density profiles are almost flat and density gradient is small compared with that of the electron temperature.

The effective heat transport coefficients are evaluated using the local density and temperature gradient by Eq. (1). Figure 7 shows the temperature dependency of the $\chi_{\text {eff }}$ at $\mathrm{r} / \mathrm{a}=0.75$ ( $r / a=0.65$ for the $R_{a x}=3.9 \mathrm{~m}$ configuration). We can see no clear difference between the values in the $R_{a x}=3.53 \mathrm{~m}$ and $3.60 \mathrm{~m}$ configurations around $3 \mathrm{~m}^{2} / \mathrm{s}$. The values are higher in the $R_{a x}=3.75 \mathrm{~m}$ and $3.90 \mathrm{~m}$ configurations and ,also, the temperature dependencies are larger in these two configurations. 


\section{Neoclassical Transport Analysis}

The role of neoclassical transport in the low-collisionality ECH plasma is investigated using the neoclassical transport database for LHD by DCOM/NNW. In order to construct the neoclassical transport database we evaluate a mono-energetic local transport coefficient using DCOM (Diffusion COefficien calculator by Monte-Carlo method), in which test particle orbits are followed solving the equations of motion in Boozer coordinates and the transport coefficient is evaluated statistically from the mean square displacement of the particles. The DCOM results have been benchmarked with the other neoclassical transport analysis codes[12].

The DCOM results are collected for a wide range of collisionality, radial electric field, minor radius, and magnetic field configurations. The transport coefficient can then be obtained from the convolution of a local Maxwellian with the mono-energetic coefficient;

$$
D_{j}^{k}=\frac{4}{\sqrt{\pi}} \int D^{k}(v)\left(\frac{v}{v_{t h}}\right)^{2 j} \exp \left[-\left(\frac{v}{v_{t h}}\right)^{2}\right] \frac{d v}{v_{t h}}
$$

where $D^{k}$ is the mono-energetic diffusion coefficient, $k=\mathrm{e}, \mathrm{i}$ (for electrons or ions) and $\mathrm{j}=1$, 2, 3. A fitting method assuming the common analytical relations depending on each collisionality regimes with their matching condition, has been used. However, this technique can not accurately describe the over lapping of the separate collisionality regimes. We apply the neural network method to interpolate the data points and construct a neural network database of the neoclassical transport coefficients for LHD. A relatively good fitting quality is obtained by the neural network method[10].

We analyze the neoclassical transport using the DCOM/NNW to show the role of neoclassical transport. In this experiment there is no measurement of the ion temperature 
profile, $\operatorname{Ti}(\mathrm{r})$, and the radial electric field, $E_{r}$. So, as a first step, we evaluated the neoclassical transport coefficient assuming $\mathrm{Ti}(\mathrm{r})=\mathrm{Te}(\mathrm{r}) / 2$, because the central ion temperature measurement by Ar spectrum indicates roughly $\mathrm{Ti}(0) \sim \mathrm{Te}(0) / 2$. We also assume the ambipolar condition for electron and ion particle flux to determine $E_{r}$ values. In this analysis the total heat flux is used to compare the experimental results with the neoclassical theory predictions, since the ion and electron heat transport coefficients depends on the ion temperature, which was not measured. The neoclassical heat flux is evaluated using the diffusion coefficients by $\mathrm{DCOM} / \mathrm{NNW}$ as

$$
Q_{k}=-n T_{k} D_{2}^{k}\left\{\frac{n^{\prime}}{n}-\frac{q_{k} E_{r}}{T_{k}}+\left(\frac{D_{3}^{k}}{D_{2}^{k}}-\frac{3}{2}\right) \frac{T_{k}^{\prime}}{T_{k}}\right\} .
$$

Figure 8 shows the total radial heat flux at $\mathrm{r} / \mathrm{a}=0.5$ as a function of the electron temperature; a) $R_{a x}=3.53 \mathrm{~m}$, b) $R_{a x}=3.6 \mathrm{~m}$, c) $R_{a x}=3.75 \mathrm{~m}$ and d) $R_{a x}=3.9 \mathrm{~m}$. The experimental total heat flux is equal to the ECH heating power, $0.88 \mathrm{MW}$. Closed circles show the heat flux of the ion-root and closed squares are the heat flux of the electron-root. The ion neoclassical heat fluxes are about half of the electron ones.

In helical systems the ambipolar condition normally has one root (ion root) or three roots (ion root, electron root and unstable root). When the electron root is realized, the large positive radial electric field reduces the transport coefficients of both ions and electrons and the neoclassical confinement is significantly improved.

We can see the electron root solutions in the $R_{a x}=3.6 \mathrm{~m}, 3.75 \mathrm{~m}$ and $3.9 \mathrm{~m}$ configurations when the electron temperature increases. In these configurations the temperature dependencies of total heat flux in the ion root are larger than that of the $R_{a x}=3.53 \mathrm{~m}$ configuration. The increase of the neoclassical transport coefficient by $1 / \nu$ helical ripple transport appears less collisional region and the lower temperature dependence would be 
observed in the optimized configuration. After the strong increase of the heat flux the solution bifurcates to two solutions in the less optimized configurations. In Fig. 4 we found the increases of Te at $n_{e} \leq 0.5 \times 10^{19} \mathrm{~m}^{-3}$ and those increases of temperature would be related to the bifurcation from the ion root to the electron root.

In all configurations the neoclassical heat fluxes become more than half of the experimental values at the highest temperatures. Especially in the less optimized configurations, the total heat flux is comparable to the experimental values if we assume the ion root. This indicates that the neoclassical transport would play a significant role to determine the achievable temperature because of the strong temperature dependency of the neoclassical heat transport coefficient, $\chi_{\text {eff }} \propto T_{e}^{7 / 2}$, for the ion root. Consequently, it is found that the neoclassical transport plays a significant role in the heat transport in the low density region.

We, next, evaluate the total radial heat fluxes near the edge region (r/a 0.75). Figure 9 shows the total radial heat flux at $\mathrm{r} / \mathrm{a}=0.75\left(0.65\right.$ in the $R_{a x}=3.9 \mathrm{~m}$ configuration $)$ as a function of the electron temperature. The experimental total heat flux is again ECH heating power, $0.88 \mathrm{MW}$. It is found that the estimated values by the neoclassical transport are smaller than the experimental values of the non-optimized configurations $\left(R_{a x}=3.75 \mathrm{~m}\right.$ and $\left.3.9 \mathrm{~m}\right)$ and that no electron root solution can be seen. This indicates that the anomalous transport dominates the heat transport at the edge region in the neoclassical transport optimized configuration.

\section{Conclusion}

We have investigated the electron heat transport in the low collisionality ECH plasma $\left(n_{e} \leq 1.0 \times 10^{19} \mathrm{~m}^{-3}\right)$ changing magnetic configurations $\quad\left(R_{a x}=3.45 \mathrm{~m}, 3.53 \mathrm{~m}\right.$ and $3.6 \mathrm{~m}, 3.75 \mathrm{~m}$, 
3.9m) in LHD. The neoclassical transport analysis shows that the neoclassical transport strongly depends on the magnetic axis position and that we obtain a neoclassical transport optimized configuration with the magnetic axis position, $R_{a x}=3.53 \mathrm{~m}$.

The effect of neoclassical transport optimization on the global energy confinement has been studied. We have found that a clear configuration dependency of the global energy confinement enhancement factors to ISS95 scaling law and that a higher enhancement factor has been observed in the neoclassical transport optimized configuration than in the other less optimized ones. The enhancement factor shows an inverse dependence on the effective helical ripple. This tendency is consistent with the recent global confinement study in stellarators[13], where the effective helical ripple dependency of the global confinement time has been shown including the experimental results by several helical devices.

The local heat transport analysis has shown that a higher electron temperature and a lower heat transport coefficient have been observed in the neoclassical transport optimized configuration $\left(R_{a x}=3.53 \mathrm{~m}\right)$ at half of minor radius, while no clear difference can be seen in the electron temperature and the heat transport coefficient near the edge region.

The comparisons of heat transport with the neoclassical transport estimated by DCOM/NNW have shown that the neoclassical transport plays a significant role in the heat transport and that the neoclassical transport optimization is effective in improving the plasma confinement in the low-collisionality LHD plasma.

\section{Acknowledgments}

This work was supported by Grant-in-Aid from the Japanese Ministry of Education, Culture, Sports, Science and Technology. Also this work is performed with the support and under the 
auspices of the NIFS Collaborative Research Program NIFS03KLHH001. 


\section{References}

[1] H.E. MYNICK, T.K. CHU, and A.H. BOOZER, "Class of Model Stellarator Fields with Enhanced Confinement", Phys. Rev. Lett. 48 (1982) 322.

[2] S. MURAKAMI, et al., "The Neoclassical-Transport-Optimized Configuration of the LHD",

Nucl. Fusion, 42 (2002) L19.

[3] S. MURAKAMI, et al., "Study of Energetic Particle Confinement in Strongly Inward Shifted Configuration of LHD",

J. Plasma Fusion Res. SERIES, Vol. 5, (2002) 620.

[4] S. MURAKAMI, et al., " Effect of Neoclassical Transport Optimization on Energetic Ion Confinement in LHD ",

Fusion Sci. Technl. 46 (2004) 241.

[5] H. YAMADA, et al., "Configuration flexibility and extended regimes in Large Helical Device", Plasma Phys. Control. Fusion, 43 (2001) A55.

[6] H. YAMADA, et al., " Energy confinement and thermal transport characteristics of net current free plasmas in the Large Helical Device ", Nucl. Fusion, 41 (2001) 901.

[7] J. MIYAZAWA, et al., "Temperature dependence of the thermal diffusivity in high-collisionality regimes in the large helical device" Plasma Phys. Control. Fusion, 47 (2005) 801.

[8] S. MURAKAMI, et al., " A Demonstration of Magnetic Field Optimization in LHD ", in Fusion Energy 2002 (Proc. 19th Int. Conf., Lyon, 2002) IAEA, Vienna (2003) CD-ROM file EX/C5-3.

[9] A. WAKASA, et al., "Monte Carlo Simulations Study of Neoclassical Transport in Inward Shifted LHD Configurations",

J. Plasma Fusion Res. SERIES, Vol. 4, (2001) 408.

[10] A. WAKASA, et al., " Development of a Neoclassical Transport Database by Neural Network Fitting in LHD ",

J. Plasma Fusion Res. SERIES, Vol. 6 (2004) 203.

[11] U. STROTH, et al., "Energy confinement scaling from the international stellarator database", Nucl. Fusion 36 (1996) 1063. 
[12] C.D. Beidler, et al.,"Neoclassical Transport in Stellarators -Results from an International Collaboration", 30th EPS Conf. Plasma Phys, Contr. Fus., St Petersburg, Russia, 2003, http://epsppd.epfl.ch/StPetersburg/pdf/P3_002.pdf.

[13] H. YAMADA, et al., "Study on magnetic configuration effects on energy confinement innet-current free plasmas based on extended international stellarator database", Nucl. Fusion 45 (2005) 1684.

\section{Figure Captions}

Fig. 1 Effective helical ripple at $r / a=2 / 3$ as a function of magnetic axis position in major radius in LHD evaluated by DCOM.

Fig. 2 Typical time development of the ECH plasma discharge in LHD ( $\mathrm{Rax}=3.65 \mathrm{~m})$; ECH heating power (top), line averaged density (center) and stored energy (bottom).

Fig. 3 Enhancement factor of the global energy confinement time relative to the ISS95 scaling law as a function of the line averaged density for five configurations of LHD.

Fig. 4 Plots of the electron temperature at $r / a=0.5$ as a function of the line averaged density for four LHD configurations; a) $\left.R_{a x}=3.53 \mathrm{~m}, \mathrm{~b}\right) R_{a x}=3.60 \mathrm{~m}$, c) $R_{a x}=3.75 \mathrm{~m}, \mathrm{~d}$ ) $R_{a x}=3.90 \mathrm{~m}$.

Fig. 5 Plots of the effective heat transport coefficient at $r / a=0.5$ as a function of the electron temperature for four LHD configurations; a) $R_{a x}=3.53 \mathrm{~m}$, b) $R_{a x}=3.60 \mathrm{~m}$, c) $R_{a x}=3.75 \mathrm{~m}$, d) $R_{a x}=3.90 \mathrm{~m}$.

Fig. 6 Plots of the electron temperature at $r / a=0.75$ ( 0.65 for the $R_{a x}=3.90 \mathrm{~m}$ configuration) as a function of the line averaged density for four LHD configurations; a) $R_{a x}=3.53 \mathrm{~m}$, 
b) $R_{a x}=3.60 \mathrm{~m}$, c) $\left.R_{a x}=3.75 \mathrm{~m}, \mathrm{~d}\right) R_{a x}=3.90 \mathrm{~m}$.

Fig. 7 Plots of the effective heat transport coefficient at $r / a=0.75\left(0.65\right.$ for the $R_{a x}=3.90 \mathrm{~m}$ configuration) as a function of the electron temperature for four LHD configurations; a) $R_{a x}=3.53 \mathrm{~m}$, b) $R_{a x}=3.60 \mathrm{~m}$, c) $\left.R_{a x}=3.75 \mathrm{~m}, \mathrm{~d}\right) R_{a x}=3.90 \mathrm{~m}$.

Fig. 8 Plots of the total heat flux of neoclassical transport evaluated by DCOM at $r / a=0.5$ as a function of the electron temperature for four LHD configurations; a) $R_{a x}=3.53 \mathrm{~m}$, b) $R_{a x}=3.60 \mathrm{~m}$, c) $\left.R_{a x}=3.75 \mathrm{~m}, \mathrm{~d}\right) R_{a x}=3.90 \mathrm{~m}$.

Fig. 9 Plots of the total heat flux of neoclassical transport evaluated by DCOM at $r / a=0.75$ ( 0.65 for the $R_{a x}=3.90 \mathrm{~m}$ configuration) as a function of the electron temperature for four LHD configurations; a) $R_{a x}=3.53 \mathrm{~m}$, b) $R_{a x}=3.60 \mathrm{~m}$, c) $R_{a x}=3.75 \mathrm{~m}$, d) $R_{a x}=3.90 \mathrm{~m}$ 


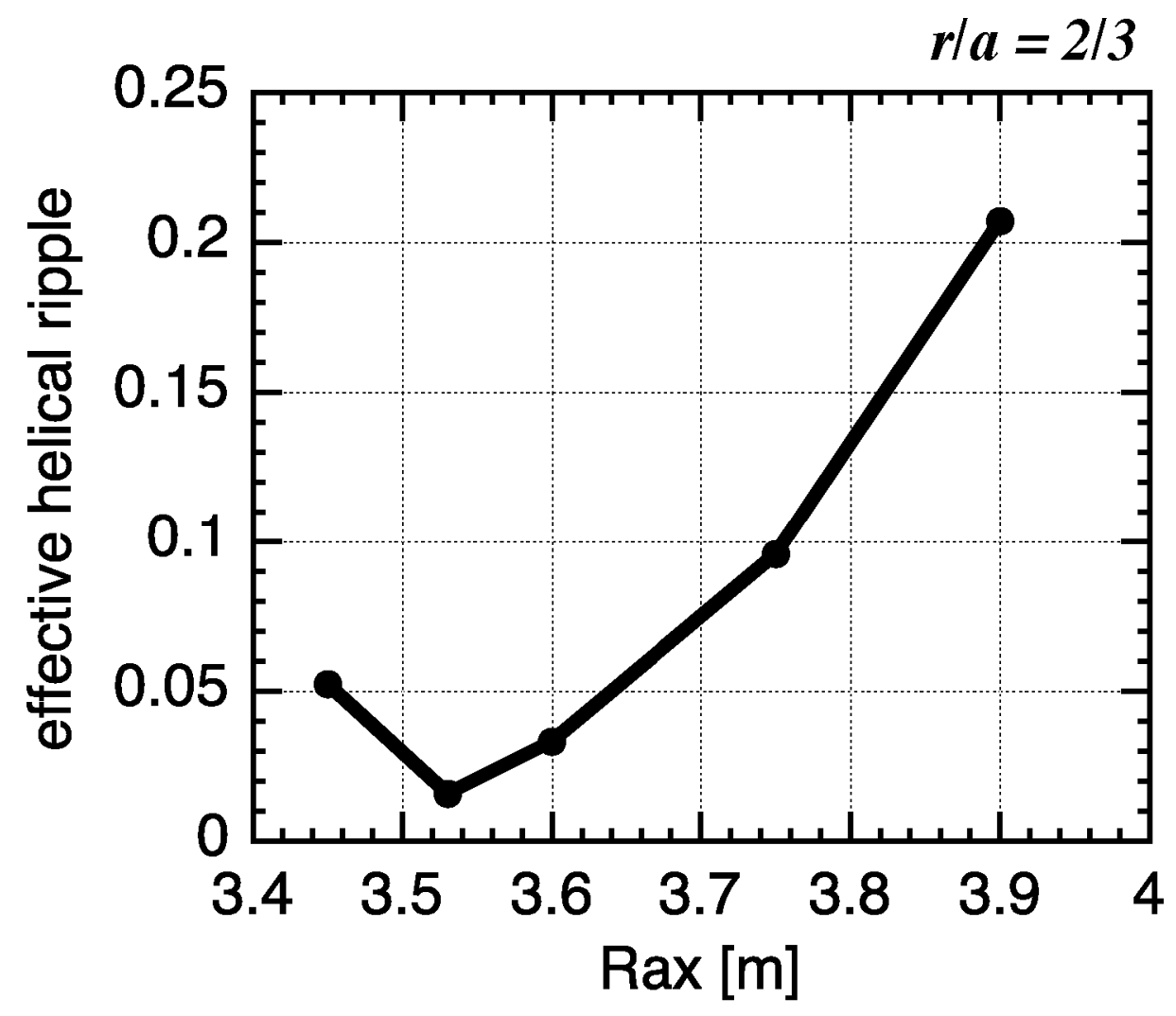

Fig. 1 Effective helical ripple at $r / a=2 / 3$ as a function of magnetic axis position in major radius in LHD evaluated by DCOM. 


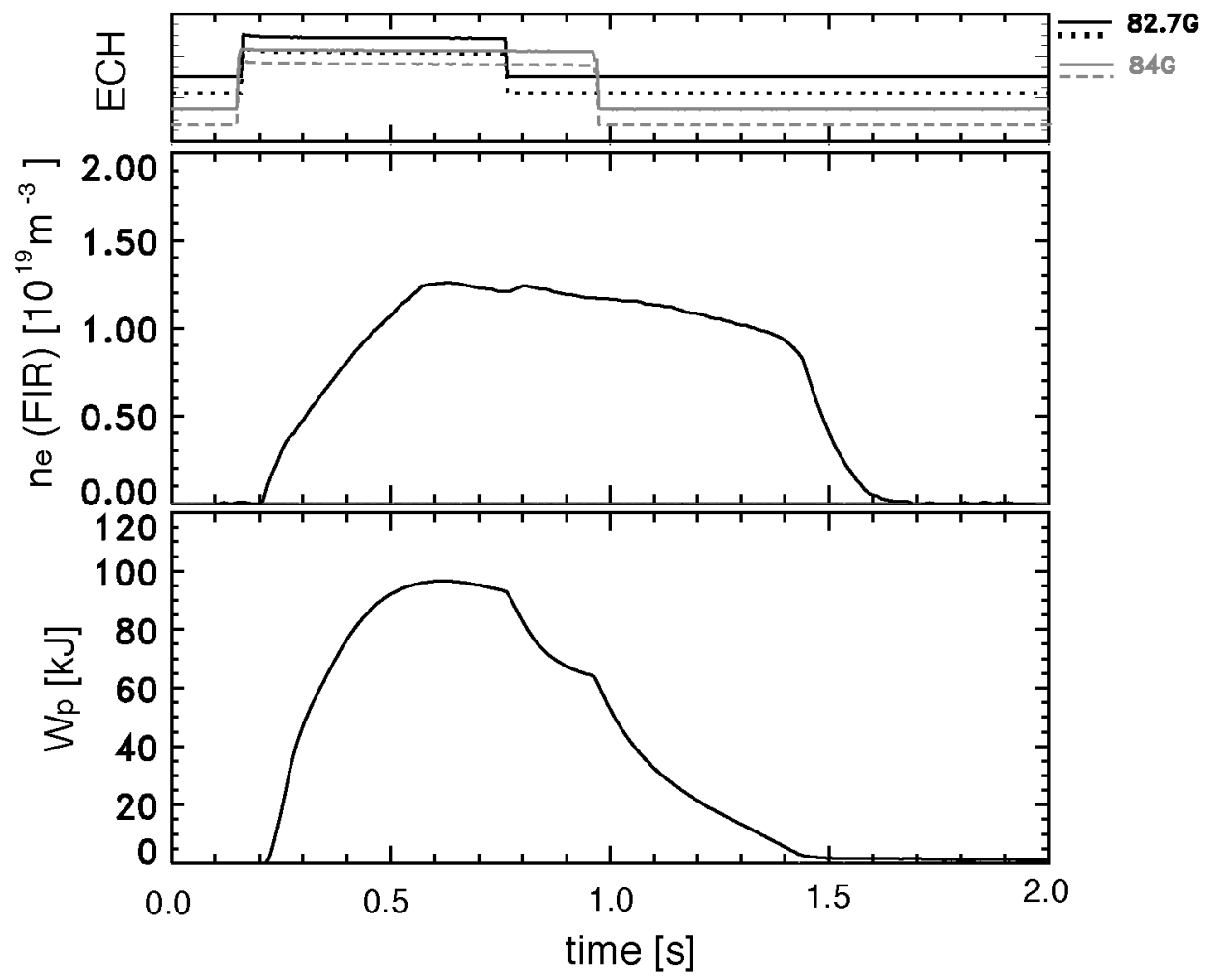

Fig. 2 Typical time development of the ECH plasma discharge in LHD ( $\operatorname{Rax}=3.65 \mathrm{~m}) ; \mathrm{ECH}$ heating power (top), line averaged density (center) and stored energy (bottom). 


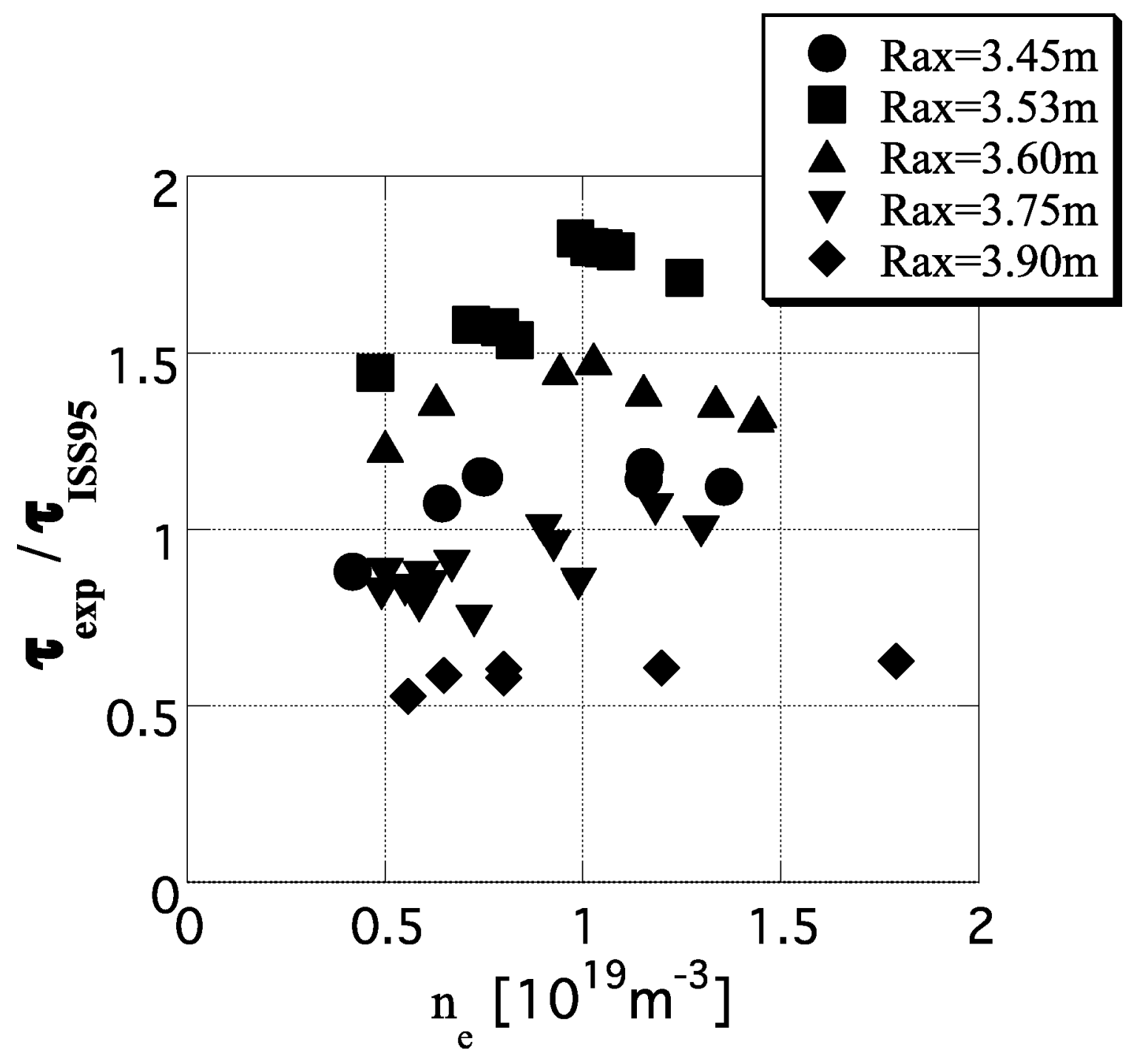

Fig. 3 Enhancement factor of the global energy confinement time relative to the ISS95 scaling law as a function of the line averaged density for five configurations of LHD. 

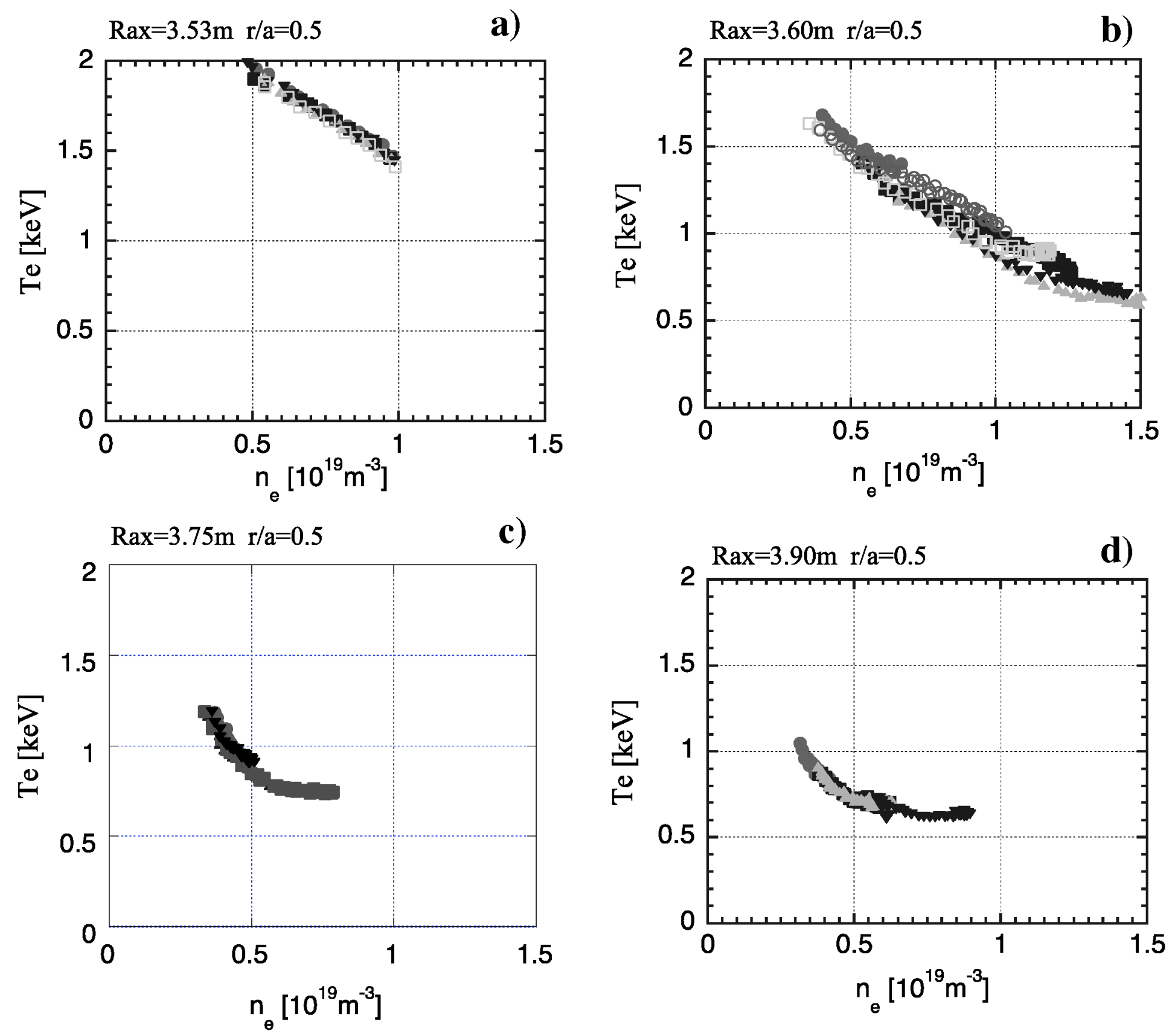

Fig. 4 Plots of the electron temperature at $r / a=0.5$ as a function of the line averaged density for four LHD configurations; a) $R_{a x}=3.53 \mathrm{~m}$, b) $R_{a x}=3.60 \mathrm{~m}$, c) $R_{a x}=3.75 \mathrm{~m}$, d) $R_{a x}=3.90 \mathrm{~m}$. 

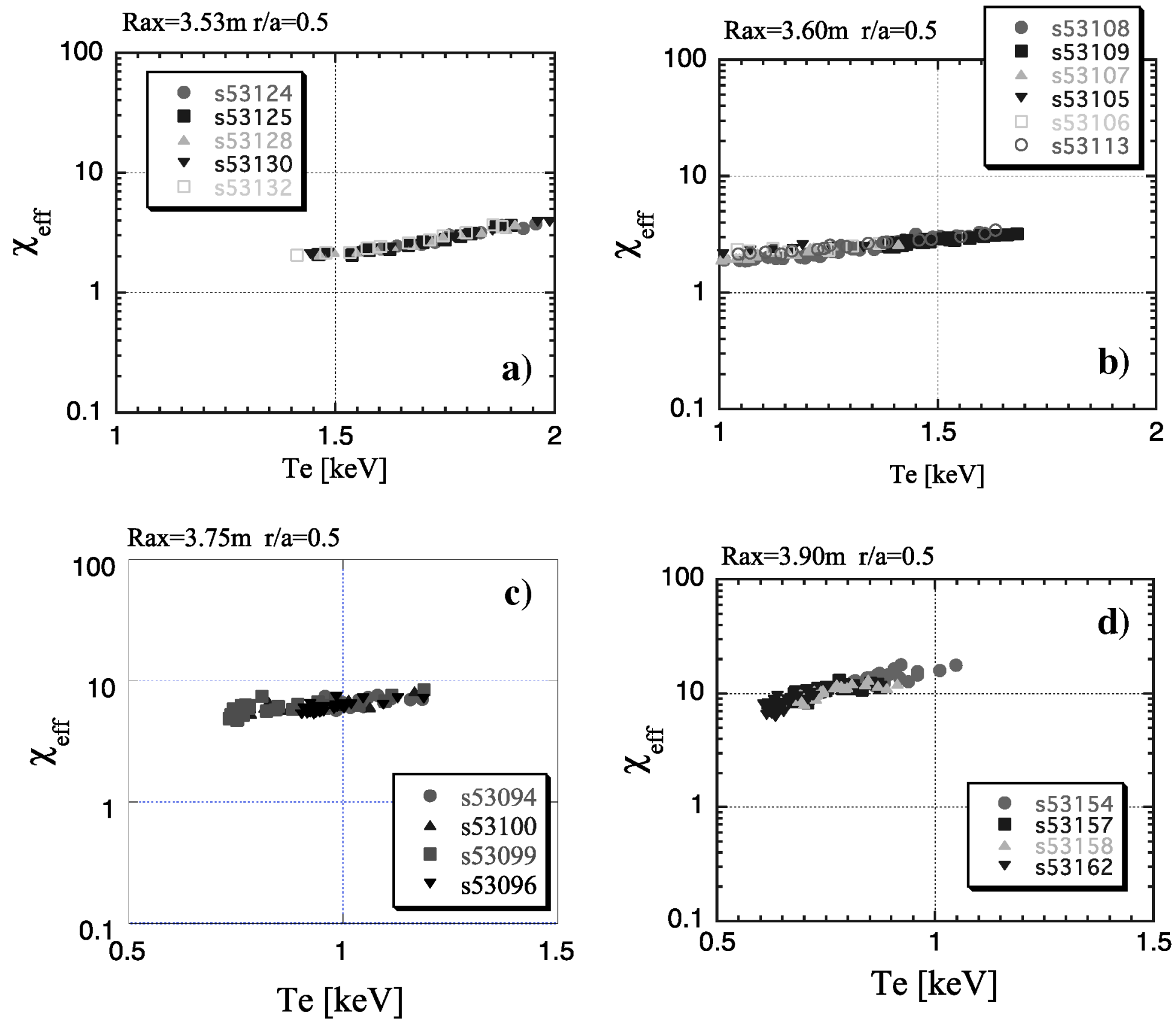

Fig. 5 Plots of the effective heat transport coefficient at $r / a=0.5$ as a function of the electron temperature for four LHD configurations; a) $R_{a x}=3.53 \mathrm{~m}$, b) $R_{a x}=3.60 \mathrm{~m}$, c) $R_{a x}=3.75 \mathrm{~m}$, d) $R_{a x}=3.90 \mathrm{~m}$. 

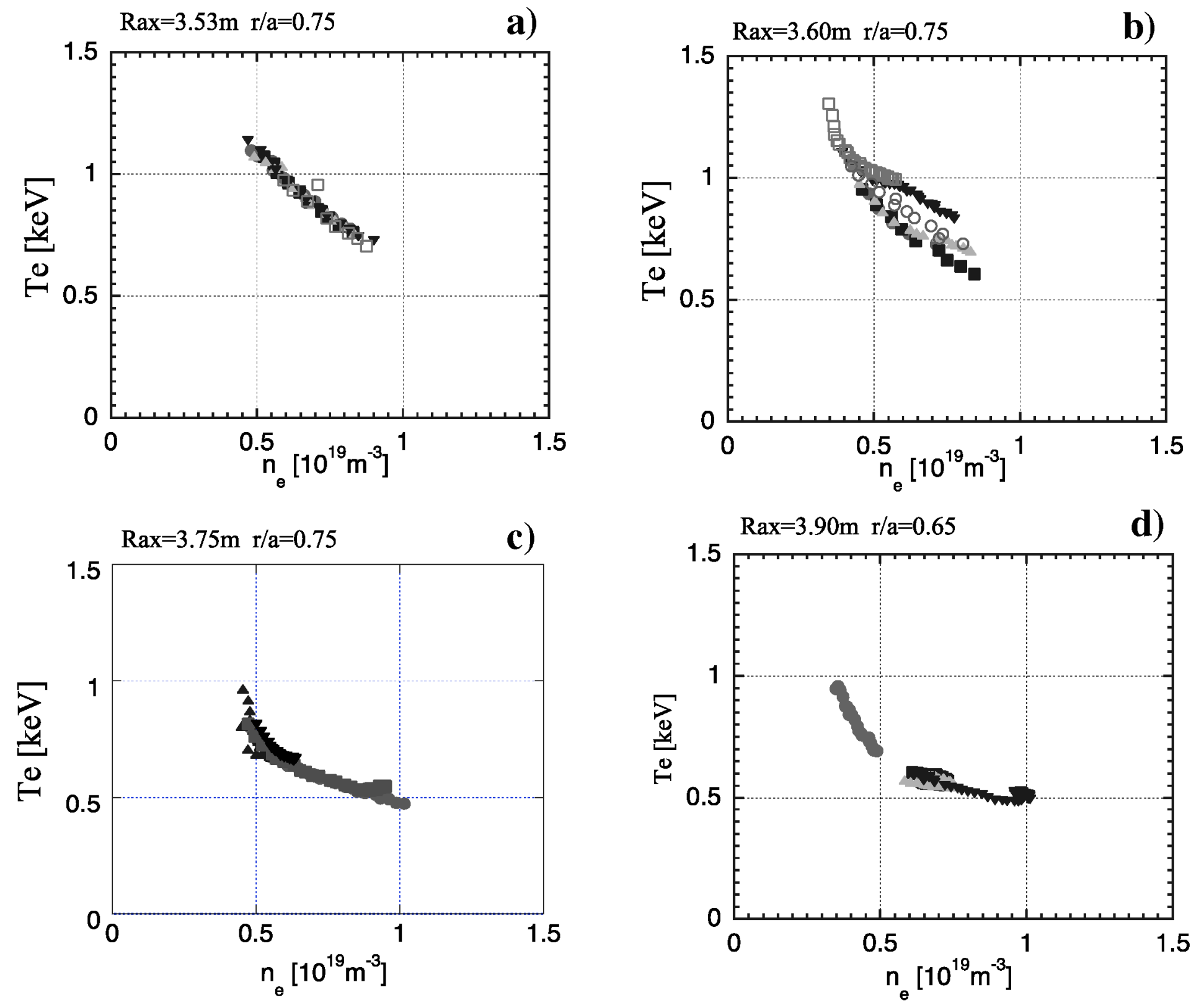

Fig. 6 Plots of the electron temperature at $r / a=0.75$ ( 0.65 for the $R_{a x}=3.90 \mathrm{~m}$ configuration) as a function of the line averaged density for four LHD configurations; a) $R_{a x}=3.53 \mathrm{~m}$, b) $R_{a r}=3.60 \mathrm{~m}$, c) $R_{a x}=3.75 \mathrm{~m}$, d) $R_{a x}=3.90 \mathrm{~m}$. 

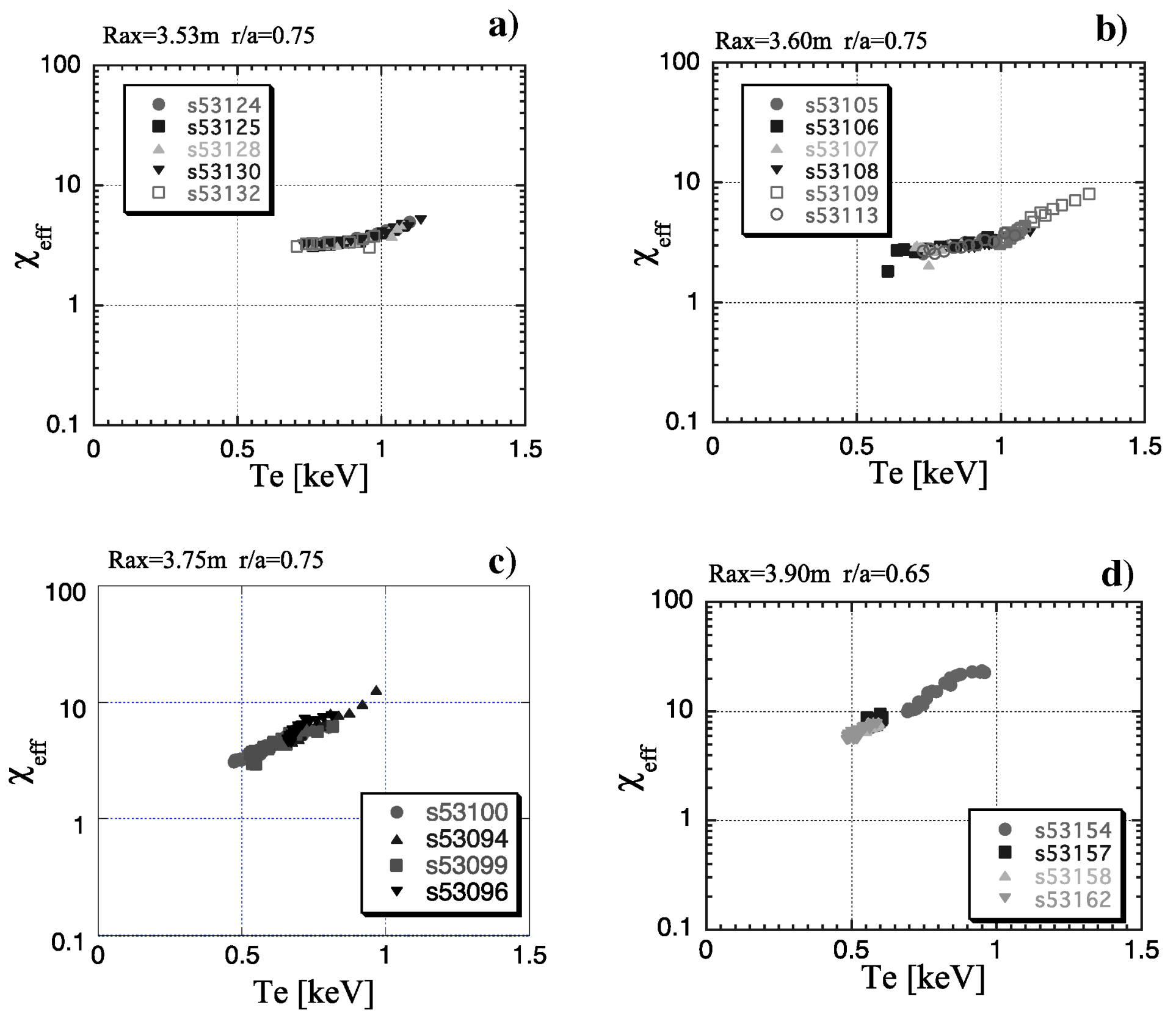

Fig. 7 Plots of the effective heat transport coefficient at $r / a=0.75$ ( 0.65 for the $R_{a x}=3.90 \mathrm{~m}$ configuration) as a function of the electron temperature for four LHD configurations; a) $R_{a x}=3.53 \mathrm{~m}$, b) $R_{a x}=3.60 \mathrm{~m}$, c) $R_{a x}=3.75 \mathrm{~m}$, d) $R_{a x}=3.90 \mathrm{~m}$. 
Total exp. flux
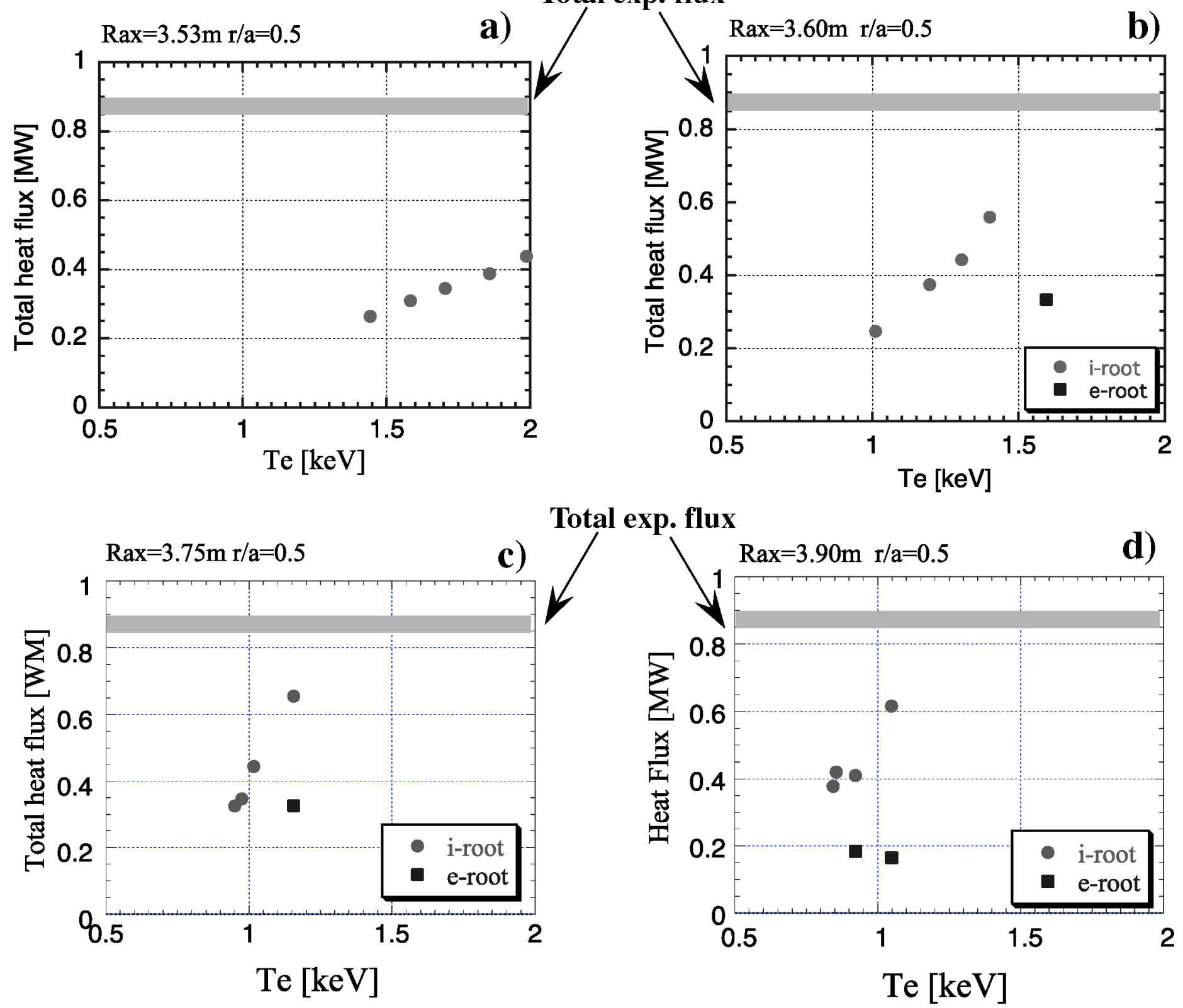

Total exp. flux

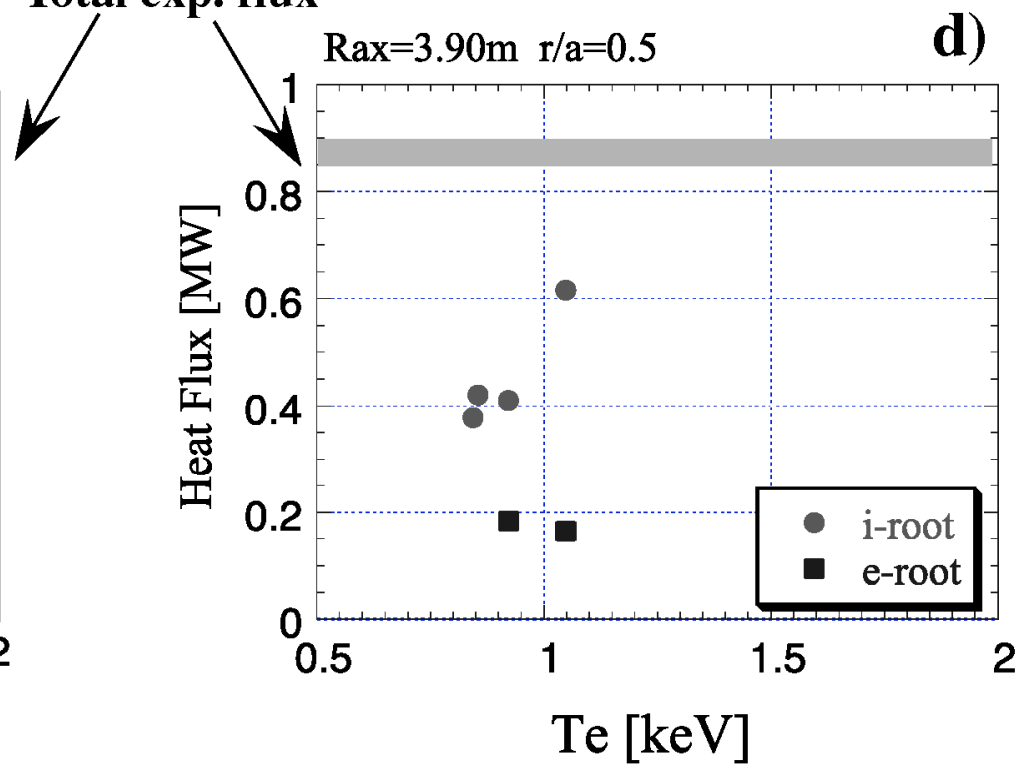

Fig. 8 Plots of the total heat flux of neoclassical transport evaluated by DCOM at $r / a=0.5$ as a function of the electron temperature for four LHD configurations; a) $R_{a x}=3.53 \mathrm{~m}$, b) $R_{a x}=3.60 \mathrm{~m}$, c) $R_{a x}=3.75 \mathrm{~m}$, d) $R_{a x}=3.90 \mathrm{~m}$. 

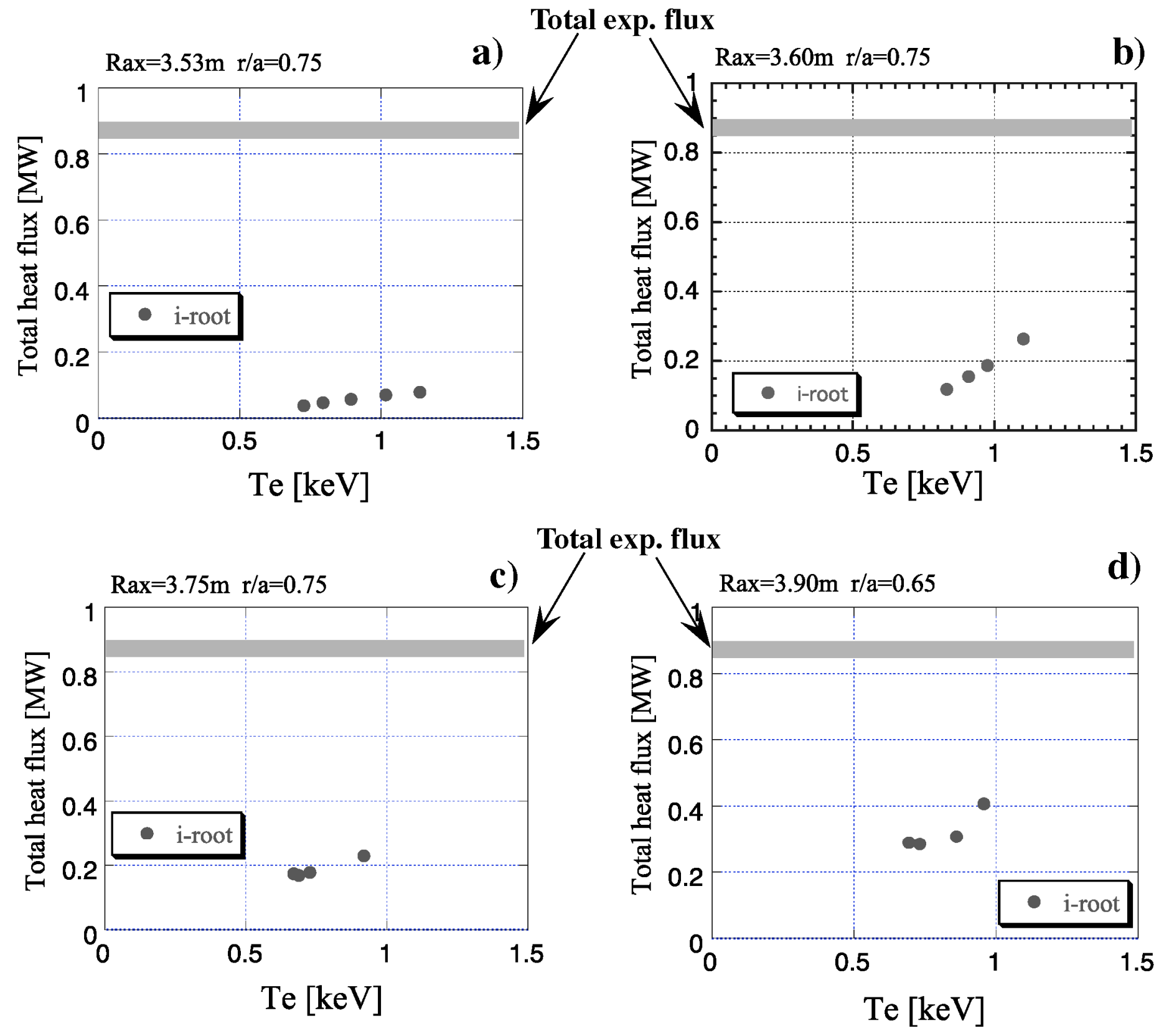

Fig. 9 Plots of the total heat flux of neoclassical transport evaluated by DCOM at $r / a=0.75$ ( 0.65 for the $R_{a x}=3.90 \mathrm{~m}$ configuration) as a function of the electron temperature for four LHD configurations; a) $R_{a x}=3.53 \mathrm{~m}$, b) $R_{a x}=3.60 \mathrm{~m}$, c) $R_{a x}=3.75 \mathrm{~m}$, d) $R_{a x}=3.90 \mathrm{~m}$ 